Reply to the Comment on "A scratch-guide model for the motion of a curling rock"

\title{
Reply to the Comment on "A scratch-guide model for the motion of a curling rock"
}

\author{
A. Raymond Penner
}

Department of Physics, Vancouver Island University, 900 Fifth Street, Nanaimo, British Columbia, Canada V9R 5S5

\begin{abstract}
Several issues were raised in the Comment on "A scratch-guide model for the motion of a curling rock." A reply to these comments is provided.
\end{abstract}

\section{Introduction}

A completely new hypothesis for why a curling rock curls was presented by Nyberg et al [1]. The hypothesis stemmed from measurements taken on ice pebbles after a curling rock had passed over them which show that the running band of a curling rock scraps the tops of the ice pebbles leaving behind scratches as deep as the asperities themselves. The hypothesis that followed is that when the asperities around the back half of the running band crossed, at an angle, the scratches formed by the asperities around the front half of the running band, a lateral force would be exerted. Experiments with curling rocks travelling on ice that was pre-scratched, using curling brooms with emery paper attached, added support to their hypothesis. It was further argued that this lateral force would be expected to be independent of the angular velocity of the curling rock which, of course, is what is found in the game of curling. Separate from the hypothesis itself, the experimental results of Nyberg et al are a step forward in the quest to find out why a curling rock curls.

The goal of the manuscript presented by Penner [2] was to provide a model to go along with Nyberg et al's hypothesis. It was concluded in this work that Nyberg et al's scratch-guide mechanism, as an explanation for the curl of a curling rock, is indeed plausible. Lozowski et al have subsequently commented on this paper. Several of these comments relate to the limiting case of $\phi \rightarrow 0$, i.e. corresponding to the curling rock not rotating, where they indicate that the model would seem to make no sense. Other comments relate to the model, seemingly ignoring certain ice parameters, having too many parameters for the fits to experimental results, and not providing physical justification for a certain approximation used in the model. One of their comments, although not referring directly to the Penner model, is the one of most interest as it refers to recent results which seem to invalidate the scratch-guide mechanism.

\section{Replies to the comments}

The comments numbered 3, 4, and 6 in Lozowski et al's list, relate to the limiting case in the model where $\phi \rightarrow 0$. I believe that the authors are mistaken as to what $\phi \rightarrow 0$ refers to. My responses to these three specific comments are: 
Reply to the Comment on "A scratch-guide model for the motion of a curling rock"

3. The time $T_{2}$ as given by equation ( $8 \mathrm{~b}$ ) corresponds to the total time it takes the disc to cross a scratch. The limit of $\phi \rightarrow 0$ corresponds to the disc travelling parallel to the scratch and therefore it will never cross the scratch, as such as $\phi \rightarrow 0$ the time $T_{2} \rightarrow \infty$. Of course as the top of the pebble has a finite diameter, $\mathrm{T}_{2}$ will have an upper limit, i.e. the time it takes an asperity to cross the top of a pebble. As stated in the paper:

"The model will break down if $T_{2}$ as given by $(8 b)$ is greater than $t_{\text {cross. }}$ In this case a trailing asperity does not fully cross a scratch."

The lower limit of $\phi$ in the model is determined by the requirement that a scratch formed by the front half of the running band is fully crossed by an asperity located on the back half of the running band. This will approximately be given by $\tan ^{-1}(2 \cdot \mathrm{scratch}$ width/diameter of a pebble). For an average pebble diameter of $4 \mathrm{~mm}$ and an average scratch width of $50 \mu \mathrm{m}$ ( Nyberg et al [1]), the lower limit of $\phi$ is therefore approximately $1.4^{0}$. As such the model does apply to typical curling situations. I do not understand where the numbers of $10^{\circ}$ and $20^{\circ}$ come from in the comment.

4. Extrapolating the model to $\phi \rightarrow 0$ does not correspond to the disc travelling parallel to but outside of the scratch wall. As explicitly stated in the paper:

"In this model the maximum lateral force occurs when the angle $\phi$ is equal to 0. This corresponds to the case where the asperity is travelling parallel to the given scratch but with half of it imbedded in the ice."

Having half of the asperity imbedded in the ice would definitely correspond to the case of maximum lateral force. Given that in this case the disc would also never cross the scratch, i.e. $\mathrm{T}_{2} \rightarrow \infty$, one would get an infinite lateral force. Of course, again, as the pebble has a finite diameter, $\mathrm{T}_{2}$ will have an upper limit. However, as stated in the paper, the model will break down if a trailing asperity does not fully cross a scratch.

6. As per my reply in 4 , extrapolating the model to $\phi \rightarrow 0$ corresponds to the disc travelling parallel to the scratch with half of it imbedded in the scratch as it crosses a pebble. The lateral force would not go to zero in this case but would be a maximum. The analogy with a knife shaving ice is not really applicable. I don't understand why the maximum lateral force would be expected to occur at around $\pi / 4$.

Comments 2 and 7 relate to certain ice parameters not being specified. My responses to these two points are as follows:

2. Independent of the complexity of the interaction between an asperity and the ice, the net result would include a pressure distribution acting along the surface of the asperity. The model of the distribution presented in Figure 2 does not seem implausible. The important point as far as the model is concerned is that the force acting on a point on the asperity will in general have components both along $\mathrm{x}^{\prime \prime}$ and $\mathrm{y}^{\prime \prime}$ as given in Figure 2, the exact form of the pressure distribution is secondary to this. 
Reply to the Comment on "A scratch-guide model for the motion of a curling rock"

The value of the parameter $\sigma_{\mathrm{o}}$ is not needed in the model. As given by equations (18b) and (19b), both components of the total force acting on an asperity are directly proportional to the value of $\sigma_{0}$. It is the ratio of these components which is of importance in the model so the value of $\sigma_{\mathrm{o}}$ falls out. Another way of understanding this is that independent of the value of $\sigma_{\mathrm{o}}$, in the game of curling a curling rock is thrown by the curler so that it travels $28 \mathrm{~m}$ down the ice sheet. The curler adjusts the launch speed to account for the ice conditions. The ice conditions have no impact on this distance. Just as the value of $\sigma_{0}$ makes no difference to the distance a rock travels down the ice in the game, in the model the value of $\sigma_{\mathrm{o}}$ makes no difference to how much a rock curls as it travels $28 \mathrm{~m}$ down the ice.

7. Again as with point 2 , both the tangential force acting on a curling rock and the lateral force acting on the curling rock are directly proportional to $\mathrm{N}_{\mathrm{a}}$. The ratio of the forces are therefore independent of $\mathrm{N}_{\mathrm{a}}$. It is this ratio that is of importance in the model. The amount a curling rock curls is proportional to the total distance a curling rock travels down the ice.

Many issues are raised with comment 8 . Primarily though this comment is indicating that there are too many parameters used to fit the model to the data. My response to this point is:

8. The initial conditions of the curling rock were not chosen to provide a good fit. The initial conditions follow from the experimental data. I do not understand why the author's claim that the initial conditions were chosen to provide a good fit, as the data used was theirs?

With regards to a difference of $30 \%$ between the friction values found at different rinks not being plausible, I refer the authors to their own work. Jensen and Shegelski [3] found that the curling rock used for their measurements curled $0.78 \mathrm{~m}$ while Lozowski, Shegelski, et al [4] found that the curling rock used for their measurements curled $1.21 \mathrm{~m}$. These measurements were done at two different rinks in Canada. Hattorri et al [5] found that curling rocks curled between $0.9 \mathrm{~m}$ to $1.4 \mathrm{~m}$ at a curling rink in Japan. The amount that a curling rock curls most definitely can vary by $30 \%$ or more at different rinks. In addition, expert curlers specifically measure the time it takes for a curling rock to travel the length of the ice to account for the significant frictional differences. For what is referred to as fast ice, where the rocks need to be thrown slower, times can exceed 25 seconds, while for slow ice the times can be below 18 seconds. There are certainly differences on the order of $30 \%$ once again which fundamentally are related to frictional differences on different ice surfaces.

With regards to point 5, as I do not understand this comment, I have no reply.

Comment 9 states that no physical justification is offered for neglecting the lateral force as the disc crosses the leading scratch wall. As evidence for this the authors use the following sentence fragments from the article; " ... the lateral force due to the disc crossing the leading scratch wall will therefore be neglected in the model ..." and "it would be expected".

9. The full section of what was written is in the article is:

"The forces acting during the two stages are symmetric and in opposite directions and would be expected to lead to there being no lateral force. However, if the asperity is conical or even 
Reply to the Comment on "A scratch-guide model for the motion of a curling rock"

irregularly shaped, it would be expected that as the asperity first makes contact with the leading scratch that the ice wall would fracture in the immediate vicinity of the initial contact point. As the neighboring sections of the asperity then pass through the wall the pressure exerted by the wall on these sections would be expected to be greatly reduced. Overall the pressure exerted on a given disc by the ice during the stage shown in Figure 3 a would be expected to be much less than the pressure exerted on the disc as it passes into the far wall. As an approximation the lateral force due to the disc crossing the leading scratch wall will therefore be neglected in the model."

The authors of the comment may believe my physical explanation is inadequate but to take sentence segments out from my explanation so as to indicate that no physical justification at all was given seems quite extreme. I will however agree that the approximation of neglecting the lateral force due to the disc crossing the leading scratch wall is probably the weakest link of the model.

The recent results as described in comment 1 are of the most interest. Although I am in disagreement with the other comments (ignoring comment 5) these experimental results seem to be in direct contradiction to the experimental results of Nyberg et al and the model that I provided to go along with the scratch-guide hypothesis. Although, in the case of Nyberg et al the surface was scratched by brooms equipped with emery paper while for Shegelski and Lozowski [6] the surface is scratched by dragging a curling rock along the ice, it may be expected that the nature of the scratching would be different. It is still confusing though for the results of Nyberg et al clearly show that a curling rock by itself severely scratches the tops of the pebbles, as can be seen in their Figure 5b. Why scratching the pebble tops with a curling broom attached with emery paper would have such an extreme effect on the curl of a curling rock, while the scratching by the textured running band of a curling rock would have no effect at all is somewhat puzzling. However, given these recent experimental results, the scraping and scratching of the pebble tops in the two cases must be very different. Ideally measurements of the tops of the pebbles before and after being scratched the two ways, in line with the measurements that were carried out by Nyberg et al, could be carried out in order to make clarify.

\section{Conclusion}

It would appear that, as of now, we still do not know why a curling rock curls. From the recent experimental results of Shegelski and Lozowski it would appear that the scratch-guide mechanism as proposed by Nyberg et al and the model provided by Penner does not explain the curl of a curling rock. The approximations made in the model and the requirement that all the frictional force must arise from the scratching in order to achieve a curl of the correct magnitude would appear to be too extreme.

However, before we throw out the baby with the bath water, the experimental results of Nyberg are most likely the key to why a curling rock curls. For if the scraping of the pebble tops makes no difference to the curl of the curling rock, why are the running bands of a curling rock specifically textured, i.e. roughened? Any model of why a curling rock curls must provide some 
Reply to the Comment on "A scratch-guide model for the motion of a curling rock"

explanation of why the running band needs to be textured in order for the rock to curl.

\section{References}

1. Nyberg, H., Alfredson, S., Hogmark, S., Jacobson, S.: The asymmetrical friction mechanism that puts the curl in the curling stone. Wear 301, 583-589 (2013b)

2. Penner, A. R.: A scratch-guide model for the motion of a curling rock. Tribology Letters 67, 35.1-35.13 (2019)

3. Jensen, E.T., Shegelski, M.R.A.: The motion of curling rocks: experimental investigation and semi-phenomenological description. Can. J. Phys. 82, 791-809 (2004)

4. Lozowski, E.P., Maw, S., Kleiner, B., Szilder, K., Shegelski, M., Musilek, P., Ferguson, D.: Comparison of IMU measurements of curling stone dynamics with a numerical model. Procedia Engineering 147, 596-601 (2016)

5. Hattori, K., Tokumoto, M., Kashiwazaki, K., et al: Proceedings of the Japanese Society of Mechanical Engineering (JSME) Symposium on Sports and Human Dynamics, Japan, October 29-31, 14, (2014)

6. Shegelski, M., Lozowski, E.: Null effect of scratches made by the curling rocks. Journal of Sports Engineering and Technology 2019; DOI: 10.1177/1754337118821575 